\section{European Spallation Source is on track}

Olof Hallonsten underestimates the status of the European Spallation Source (ESS) project and overestimates the risks to its success (Nature 518, 275; 2015).

Construction has been under way for almost six months, after Sweden and Denmark secured a combined commitment by ESS member nations to contribute more than $97 \%$ of the building costs. This commitment is underpinned by the importance of the ESS to their own national research programmes and by their prior investment in comprehensive scientific, technical and management reviews of the construction plans.

We have recruited some 300 experienced staff from around the world. More than 1,000 collaborators are bringing their collective knowledge to the project at the ESS site near Lund, Sweden, and at partner institutions in Europe.

Regarding Hallonsten's doubts about the legal and financial framework for the life cycle of the facility, a European Research Infrastructure Consortium is due to be approved this year that is set to provide such a framework.

Neither do we do deals "behind closed doors". We have engaged more than 100 independent scientific, technical and management experts to conduct routine reviews of the project. Open meetings with the scientific community are organized twice a year. There will be risks and challenges along the way, but we are firmly committed to delivering the ESS facility on time and on budget.

Jim Yeck European Spallation

Source, Lund, Sweden.

jim.yeck@esss.se

\section{City data can inform decision theory}

Data are being collected on human behaviour in cities such as London, New York, Singapore and Shanghai, with a view to meeting city dwellers' needs more effectively. Incorporating decision-making theory into analyses of the data from these 'urban observatories' would yield further valuable information.

Huge amounts of data can be amassed from digitized social, cultural and economic records of citizens' choices and behaviours, as well as from fixed and mobile sensors, including those at urban vantage points. This information allows 'natural' experiments that chronicle the population's responses as the urban environment undergoes planned and unplanned changes.

Combining such data with models of human decisionmaking can provide a deeper insight into real-world choices. For example, analysis of local crime statistics together with results of cognitive testing in pre-school children showed that a murder occurring near their homes influenced children's levels of attention and impulse control at school (P. T. Sharkey et al. Am. J. Public Health 102, 2287-2293; 2012).

Twitter messages, requests for city services and information, and other behavioural metrics could likewise be used to investigate human decision-making.

Aristides A. N. Patrinos ${ }^{\star}$ New York University, New York, USA. aap11@nyu.edu

${ }^{*}$ On behalf of 6 correspondents (see go.nature.com/mabntz for full list).

\section{Access to the bigger picture in histology}

Published histology images tend to focus on only a small part of a fixed tissue slice. Journals could overcome this limitation by asking authors to make histological data from the rest of the slide available wherever possible, to extend and complement their published micrographs.

Tools are already available to scan entire histology slides for online study by virtual microscopy (see www.histology.be). This enables histology images to be shared worldwide, for example from pathology archives (M. G. Rojo et al. Int. J. Surg. Pathol. 14, $285-305$; 2006) or to assist medical diagnostics.

Compiling the extended histology information in a public database would provide an invaluable resource for researchers. It could be used to access unexplored raw data from tissue slices, or to stimulate new analyses or alternative interpretations.

Yves Poumay, Michel Jadot University of Namur, Belgium. yves.poumay@unamur.be

\section{Scrap rainbow colour scales}

It is time to clamp down on the use of misleading rainbow colour scales that are increasingly pervading the literature and the media (see http://tiny.cc/ endoftherainbow). Accurate graphics are key to clear communication of scientific results to other researchers and the public - an issue that is becoming ever more important.

Aside from the challenge they pose for colour-blind readers (S. C. Allred et al. Nature 510, $340 ; 2014)$, spectral-type colour palettes can introduce false perceptual thresholds in the data (or hide genuine ones); they may also mask fine detail in the data (D. Borland and R. M. Taylor Comput. Graph. Appl. 27, 14-17; 2007). These palettes have no unique perceptual ordering, so they can de-emphasize data extremes by placing the most prominent colour near the middle of the scale.

These issues can have profound consequences: for example, changing to a nonrainbow colour scale improved diagnostic accuracy for heart disease (M. Borkin et al. IEEE Trans. Vis. Comput. Graph. 17, 2479-2488; 2011).

Journals should not tolerate poor visual communication, particularly because better alternatives to rainbow scales are readily available (see, for example, go.nature.com/zvouhq and http://colorbrewer2.org). We urge all journals to stipulate in their guidelines that graphics must convey accurate and accessible information.

Ed Hawkins ${ }^{\star}$ University of Reading, UK.

e.hawkins@reading.ac.uk ${ }^{*}$ On behalf of 5 correspondents (see go.nature.com/usnhfy for full list).

\section{Concept of multiple sexes is not new}

The idea that there are more than two biological sexes is not as recent as you imply (Nature 518, 288-291; 2015). It emerged in the early 1990s after feminist critics of science joined forces with an intersex activist movement. Their aim was to prevent reinforcement of the artificial two-sex construct by reforming the practice of surgical intervention (see, for example, A. Fausto-Sterling The Sciences 33, 20-24 (1993) and S. J. Kessler Lessons from the Intersexed Rutgers Univ. Press, 1998).

These groups pointed out that science is not isolated from society: ideas that stimulate understanding travel into the lab from street activists, literature and varied scholarship, and move back out again. As a result of their efforts, research scientists were pushed into visualizing the previously invisible.

Anne Fausto-Sterling Brown University, Providence, Rhode Island, USA.

anne_fausto-sterling@brown.edu

\section{CORRECTION}

In the Outlook article 'Taking a shot at protection' (Nature 516, S12-S13; 2014), the $x$ axis on the graphic 'Immunity boost' was incorrectly labelled. It should have read 'Age at livercancer diagnosis'. 